\title{
Photo 3D technology applied to e-Learning tools production for animal biology
}

\author{
Pierre-Eric Sautière ${ }^{1}$, Catherine Delbende ${ }^{1}$, Bernard Deleplanque ${ }^{2}$, Bernard \\ Mikolajczyk ${ }^{2}$, Jacopo Vizioli ${ }^{1}$ \\ ${ }^{1}$ Univ. Lille, Biology Department, Lille, France, ${ }^{2}$ Univ. Lille, Direction Innovation \\ Pédagogique, Lille, France.
}

\begin{abstract}
The teachers from the Biology Department of the University of Lille have been developing for the last two years, a digitalization program of naturalist collections. This project aims to preserve this scientific heritage and to use it for animal and plant biology teaching. Specimens are digitalized by a photo $3 D$ capture system, that produces a $360^{\circ}$ and/or hemispheric images of the objects starting from high-resolution pictures. Based on the use of this particular imaging technology, teachers realized multimedia eBooks and a series of files "at the glance" for practical works in animal biology for Bachelor students in Life Sciences. These supports, enriched by graphic complements, texts, legends and interactive animations, are available on the pedagogic platform Moodle. These digital tools are viewable on computers and can be handled on smartphones and tablets for a nomad utilisation. Students generally consider these supports useful for learning and they consult these resources before, during and after the practical sessions. This distance-learning approach gives the students a complete autonomy for practical session preparation and reviews. The innovative tools here presented constitute a useful learning complement to classical academic lectures in animal biology.
\end{abstract}

Keywords: photo 3D; eBooks; e-Learning; anatomy; animal biology. 


\section{Introduction}

Teaching of Biology in European Universities is generally based on classical academic lectures and practical sessions. The transition from the high school to the Bachelor environment requires the students more autonomy and a different way of studying. The amount of knowledge to acquire in term of vocabulary and content has consequently a negative impact on learning feedback. A group of teachers from the Biology Department of the University of Lille has been developing in the last years the usage of Information and Communication Technologies for Education (ICTEs) to improve learning and attractiveness of animal and plant biology for undergraduate students in Life Sciences. The aim is to produce student-centered innovative digital tools (videos, eBooks and photo 3D from naturalist collections) made available on the pedagogic platform Moodle for a free and unlimited access on different devices. Undergraduates can use these distance-learning resources to complete the study of face-to-face academic lectures and practical sessions content. This blended-learning approach allows them: a) to review the theoretical content of the academic lectures; b) to virtually handle the specimens of different animals illustrated during the practical sessions and c) to enlarge the knowledge on the subject through the lecture of the interactive digital tools as well as of the web sites available as hyperlinks. This project has the goal of fostering the autonomy and the comparative analysis capabilities of students as well as the exchanges between peers. In addition, the use of different devices like smartphones and tablets aims to improve their curiosity and involvement in studying biology. As an example of this e-Learning policy, we present here the production of a digital manual and a series of files for practical session in animal biology describing teeth features in relation to specific diets and skull evolution in Mammals.

\section{Materials and Methods}

\subsection{Photo 3D capture system}

The Biology Department of the University of Lille possess a naturalist collection regularly used for pedagogic purposes. Digitalization of this scientific heritage is performed using the PackshotCreator (Levallois-Perret, France) photo 3D system, equipped by five Canon EOS $700 \mathrm{D}$ digital cameras. Briefly, the shooting is performed on specimens placed on a rotating plate, which allows obtaining $360^{\circ}$ and/or hemispheric HD (18 Million Pixels) images of the objects. The size of specimens can varies from $1 \mathrm{~cm}$ to about $100 \mathrm{~cm}$. For small samples and/or details captures we used macrophotography lenses (Canon $100 \mathrm{~mm}$ Macro). A hyper focus system allows the production of perfectly sharp pictures. Images are exported under different formats (tiff or jpg) following their usage for printing, multimedia textbooks or web publication. Animations of photo 3D images are generated in HTML5 format with the 
PackshotViewer software and are viewable on all Internet browsers. Photo 3D of these naturalist collections are available on the free access website http://photo3d.univlille1.fr/360/.

\subsection{Interactive multimedia files and eBooks}

The available photo 3D images were used to produce a series of files describing teeth's features and evolution in Mammals (http://photo3d.univlille1.fr/dents_et_regimes/index.html). Teachers entirely conceived these documents. Multimedia engineers treated single images with Photoshop (Adobe) to enrich them with legends and colours displaying specific elements of the different samples. Animations were realised with the Adobe Muse software.

We recently produced several multimedia eBooks made available for students for practical session preparation and review before learning feedback. These digital supports contain text, schemes, images, photo galleries, a glossary and other interactive widgets to explain and complete the work made during the lab session. The integration of hypertext links and QR codes into the eBook gives the students the possibility to directly point to the photo 3D database described above to observe and virtually handle the different samples. These digital resources, made with free software iBooks Author distributed by Apple, can be exported in iBook or pdf format for Apple or PC/Android users, respectively. These supports are so far available in French but English versions are being programmed for our Bachelor students of the bilingual (English-French) option.

\section{Results}

The University of Lille recently acquired a 3D photo capture system aimed to digitalize its scientific heritage. The Biology Department is producing a database of plant and animal collections to be used for didactic purposes (http://pod.univ-lille.fr/video/5946-unenouvelle-vie-pour-des-collections-naturalistes-dantan/). Based on this context, a group of teachers developed a distance-learning project in animal biology for Bachelor in Life Sciences students aimed to illustrate, by digital tools, Mammals' teeth features according to their diet. The main learning objective of these innovative supports is to illustrate with texts, schemes and interactive 3D photos the set of teeth of different Mammals described during academic lectures and practical sessions. These supports are compatible with any digital device (computers, tablets or smartphones) and Internet browser. Students have access to these documents in the two weeks preceding the practical session. During the practical work, they observe the samples issued from the zoology collection and perform comparative analyses on skulls morphology/evolution as well as on the set of teeth in different animals. Finally, students use these digital resources to review before the final examination at the end of the semester. The 3D photos of selected specimens were used to 
make a series of about 30 files "at the glance“ describing the evolution, the number, the morphology and the action of teeth associated to different diets of Mammals (carnivorous, herbivorous, omnivorous,...) (Figure 1).

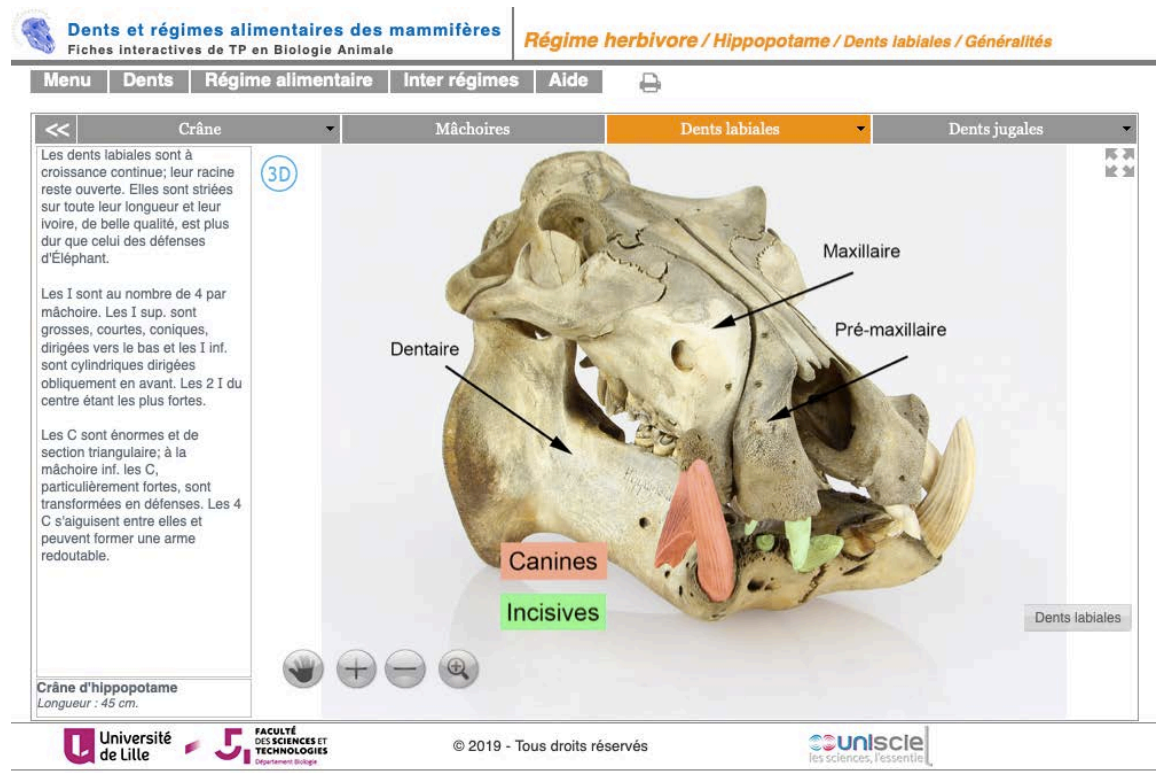

Figure1. Photo 3D of a hippopotamus skull as an example of herbivorous diet. Teeth names and positions are highlighted by coloured areas. Arrows indicate bones supporting the different types of teeth. The description of their features (quantity, shape, size,...) is shown on the left. Users can choose various tabs to see different regions of the skull and the related teeth. Mouse controlled rotation of the 3D image and the tools in the lower part of the

windows give access to different zoom levels permitting to appreciate the details of the skull through highresolution pictures. The upper menu allows navigating in the web site to observe the set of teeth and diets of other animals.

These documents are mainly intended for preparation and review of practical session on animal evolution and adaptation unit (BSci2). A questionnaire was submitted to a sample of students $(n=38)$ to have a feedback on the usage of these tools. In general, results indicated these supports as easy to use for most of them (92.1\%). The files were viewed on both computers (81.6\%) and tablets (18.4\%). Students used these digital tools to prepare the practical session (13.2\%), to review before exams (50\%) or both $(18.7 \%)$. A few of them declared the files useless for learning or redundant with the eBook content (7.9\%). Some students did not answer this question (10.5\%) or (Figure 2). 


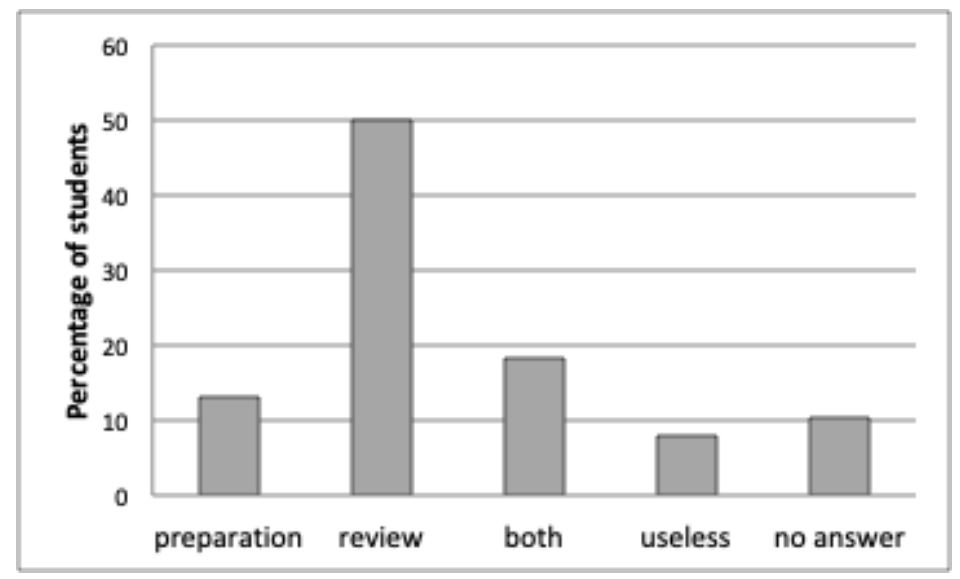

Figure 2. Digital files usage feedback. Histograms $(n=38)$ represent the percentages of students using the files on "Mammals' teeth and diet" for practical session preparation and/or reviewing. Negative answers are also reported

These files were associated to an interactive eBook containing schemes, texts, images, a glossary and hyperlinks. This multimedia textbook resumes the main content on the subject and describes the adaptation of both teeth and skull features to the diet. This interactive tool is made available for viewing and downloading on the didactic platform Moodle in iBook or pdf format for Apple and PC/Android devices, respectively. In the period tested (February-June 2018) the eBook was viewed 297 times by the 153 students concerned by the related content. Some of them complained about the loss of interactivity (image galleries, videos and other widgets) using PC or Android devices respect to Apple ones. To solve this problem, we supplied additional pictures or QR codes redirecting towards specific videos at the end of the pdf version.

\section{Discussion}

This project is included in a larger program of valorisation and didactical use of the naturalist collections of the Biology Department of the University of Lille. The aim is to restore and preserve many ancient and fragile specimens as well as developing new ways to use them for Life Sciences teaching (Cook et al,. 2014). This goal passes trough the digitalization of this scientific heritage, which we perform with a photo 3D system. This kind of image capture approach is generally exploited by private companies and is mostly intended for e-Commerce. The usage here described constitutes, to our knowledge, the first example of naturalist collections digitalization associated to didactic purposes for higher education in Life Sciences. We integrated the use of 3D photos in our academic lectures and practical session on animal biology. Students observe these samples on their digital devices without any risk of damage of this historical heritage. 
Based on the 3D images we developed a new project of files "at the glance" as a support for a practical session on teeth and diets in Mammals for Life Sciences (BSci2) students. These interactive files allow the virtual handling of specimens, favouring details viewing and anatomy knowledge. The use of these files, freely accessible on the web, can be easily extended to other university courses (eg. veterinary, educational sciences,...). In addition, because of their ease of use and pleasant presentation, they can be exploited for teeth and diet courses in middle and high school levels.

We also produced a series of multimedia textbooks in animal and plant biology for Bachelor in Life Science instruction. Students generally read and use these textbooks on their own computer or tablet. Smartphones are less used during the practical session, probably because of the reduced size of the screen that does not allow an easy viewing of images and written content. On the other hand, these mobile devices are mainly appreciated for their nomad utilisation and quick review of punctual subjects. Many students prefer downloading and printing the document: actually, during the practical session, they use the paper support to customize it with notes, printed screenshots, comments and legends. It is consistent with the report of George and collaborators indicating that the use of digital devises cannot replace printed handouts (George et al., 2013).

Taken together, practical files and the eBook are complementary resources: the first ones mainly favouring undergraduates' comparative analyses and observation skills, the second one mostly being a digital manual for the description of samples and practical session content. In addition, the eBook allows widening and consolidate the knowledge by specific inset, hyperlinks (e.g. web pages, videos) or quizzes about the treated subject. Students' feedback on these multimedia tools is positive in terms of usefulness and autonomy gain during the practical sessions. This result is consistent with the observation of Mayfield and colleagues that noticed a less seek of instructors during practical work on human anatomy in case of availability of digital supports (Mayfield et al., 2013). The production of such interactive eBooks in biology fields is at present poorly developed. Indeed, multimedia resources were just described as useful for chemistry (Jordan et al., 2016) and molecular biology (Laneuville \& Sikora, 2015) learning. The use of tablets and e-Learning strategies were reported as relevant for human anatomy teaching (Trelease, 2016; Scibora \& Mead, 2018) and other educational fields (Nguyen et al., 2015). The usage of our digital documents before (preparation), during (observation and analysis) and after (review) the practical session resulted coherent with the didactic objectives of the project. Our experience of blended learning strategy, coupling academic lectures and practical work with non-attendance-based teaching, resulted positive in term of students appreciation and learning feedback, as previously reported for human anatomy teaching (Pereira et al., 2007). This is why we wish to carry on and develop this student-centered program of eLearning tools production, which effectiveness was already established for biology 
instruction (Connel et al. 2016). In addition to face-to-face instruction, which remains a necessary way of teaching for academic education, the association of 3D photos with other multimedia tools appears as a useful approach for distance-learning and constitutes an innovative potential for biology teaching.

\section{Acknowledgements}

The project "Mammal teeth and diets" is funded by UNISCIEL (Université des Sciences en ligne, http://www.unisciel.fr). Digital textbooks were granted by calls from the University of Lille for the production of educational media resources. Authors thank Thierry Danquigny and Teodorina Tibar, educational designers from the EdTech \& Digital Pedagogy Department of the University of Lille, for their accompaniment throughout the production of these digital supports.

\section{References}

Connell GL, Donovan DA, Chambers TG. (2016) Increasing the use of student-centered pedagogies from moderate to high improves student learning and attitudes about biology. CBE Life Sci. Educ. 15:1-15. doi:10.1187/cbe.15-03-0062.

Cook JA, Edwards SV, Lacey EA, Guralnick RP, Soltis PS, Soltis DE, Welch CK, Bell KC, Galbreath KE, Himes C, Allen JM, Heath TA, Carnaval AC, Cooper KL, Liu M, Hanken J, Ickert-Bond S. (2014). Natural history collections as emerging resources for innovative education. Bioscience 64:725-734. doi:10.1093/biosci/biu096.

George P, Dumenco L, Doyle R, Dollase R. (2013). Incorporating iPads into a preclinical curriculum: A pilot study. Medical Teacher 35:226-230. doi:10.3109/0142159X.2012.735384.

Jordan JT, Box MC, Eguren KE, Parker TA, Saraldi-Gallardo VM, Wolfe MI, GallardoWilliams NT (2016). Effectiveness of Student-Generated Video as a Teaching Tool for an Instrumental Technique in the Organic Chemistry Laboratory. Journal of Chemical Education. 93:141-145. doi:10.1021/acs.jchemed.5b00354.

Laneuville O \& Sikora D. (2015). Quantitative analysis of the usage of a pedagogical tool combining questions listed as learning objectives and answers provided as online videos. Future Internet. 7:140-151. doi:10.3390/fi7020140.

Mayfield CH, Ohara PT, O’Sullivan PS. (2013). Perceptions of a mobile technology on learning strategies in the anatomy laboratory. Anatomical Science Education. 6:81-89. doi:10.1002/ase.1307.

Nguyen L, Barton SM, Nguyen LT. (2015). IPads in higher education - Hype and hope. British Journal of Educational Technology. 46:190-203. doi:10.1111/bjet.12137.

Pereira JA, Pleguezuelos E, Merí A, Molina-Ros A, Molina-Tomàs MC, Masdeu C. (2007). Effectiveness of using blended learning strategies for teaching and learning human anatomy. Medical Education. 41:189-195. doi: 10.1111/j.1365-2929.2006.02672.x. 
Scibora LM \& Mead T. (2018). The Influence of iPads on Course Performance and Student Perceptions of Learning in Human Anatomy. Journal of Teaching and Learning with Technology. 7:108-124. doi:10.14434/jotlt.v7n1.23973.

Trelease, R. B. (2016). From chalkboard, slides, and paper to e-learning: How computing technologies have transformed anatomical sciences education. Anatomical sciences education, 9(6), 583-602. doi: 10.1002/ase.1620. 